\title{
Neanatal Intestinal Obstruction
}

\author{
Mitra Khalili1," \\ ${ }^{1}$ Shahid Beheshti University of Medical Science \\ "Corresponding author: Mitra Khalili, Shahid Beheshti University of Medical Science. E-mail: m.khalili76@yahoo.com
}

Received 2016 December 21; Accepted 2017 February 08.

\begin{abstract}
Background: Intestinal obstructions are the most common surgical emergencies in the neonatal period. Neonatal intestinal obstruction occurring during the first month of life. Early and accurate diagnosis of intestinal obstruction is important for proper patient management. This presentation is a brief review of studies in Pubmed and Google scholar. For evaluation and diagnosis, intestinal obstruction in neonates can be divided into either high or low obstruction on the basis of dilated bowel loops present on abdominal radiographs. A few dilated bowel loops are seen with high intestinal obstruction and several bowel loops are generally seen with low intestinal obstruction. High intestinal obstructions are defined as occurring proximal to the ileum with differential diagnosis and radiographic appearance as below: 1) Gastric atresia: in microgastria a distended esophagus and a small midline stomach and In gastric atresia single bubble sign" with no distal gas. 2) Duodenal atresia: double bubble sign 3) Malrotation with Ladd bands and midgut volvulus can be partial or complete obstruction: the abdominal radiograph in malrotation is nonspecific. It may be normal or may show a proximal bowel obstruction pattern, or show dilatation of multiple bowel loops. 4) Duodenal web partial obstruction with small amount of distal bowel gas. 5) Annular pancreas. 6) Preduodenal portal vein. 7) Jejunal atresia: triplebubble sign with no gas in the distal bowel. Dilatation of the duodenum is a sign of chronic obstruction, therefore seen in duodenal atresia, duodenal web and annular pancreas. When obstruction occurs acutely after birth in midgut volvulus, the duodenum is not usually dilated on plain film. In complete obstruction in the high jejunum, no further roentgenographic studies are required, however, in partial obstruction (small amount of distal gas) upper GI study may be helpful to distinguish between duodenal web and malrotation with Ladd bands or midgut volvulus. The classic upper gastrointestinal appearance of malrotation with volvulus consists of an abnormal course of the duodenum that fails to cross the midline combined with a corkscrew appearance. In contrast, low intestinal obstructions involve the distal ileum or colon and typically result in diffuse dilatation of multiple small-bowel loops. If distal small bowel obstruction is suggested, a contrast enema usually is necessary. It is not possible to differentiate distal small bowel from colonic obstruction on the basis of plain abdominal roentgenograms, and consequently, contrast enema is necessary to clarify microcolon in ileal atresia and meconium ileus and differentioation from other pathology as below: 1) Meconium ileus: unused colon (i.e., microcolon), within which are multiple small filling defects representing meconium concretions. If there is reflux of contrast material beyond the ileocecal valve, multiple small filling defects (meconium concretions) also may be seen in the terminal ileum. 2) Ileal atresia: Microcolon and blind ending ileum on contrast enema. 3) Meconium plug and small left colon syndrome: multiple filling defects (i.e., meconium plugs) is seen in splenic flexure with left sided microcolon and with normal rectum. 4) Hirschsprung disease: abnormal rectosigmoid ratio $(<1)$, transition zone of rectal narrowing, irregular rectal contraction and retained contrast material on delayed radiographs, 5) Colonic atresia: contrast enema study typically shows a distal unused colon (i.e., microcolon), with the more proximal markedly dilated colon ending in a blind pouch.6) Anorectal malformation. In any of these cases, if intestinal perforation occurs meconium peritonitis can result and peritoneal calcifications can be seen on plain films.

Conclusions: Intestinal obstructions are the most common surgical emergencies in newborn infants which require early and accurate diagnosis. An understanding of the characteristic imaging appearance of various causes of neonatal bowel obstruction on abdominal X-ray can lead to correct diagnosis or leading a guide to the next appropriate step. After abdominal X-ray that shows the presence of a neonatal high intestinal obstruction, an upper gastrointestinal series is typically performed for further evaluation. However, neonates with classic radiographic findings of high intestinal obstruction, such as duodenal atresia, may undergo surgery without any additional imaging study. An enema examination is used for further investigation of low intestinal obstruction in neonates.
\end{abstract}

Keywords: Neonate, Bowel Obstruction, Radiography, Contrast Enema, Upper GI Series

This is an abstract presented in the 33rd Iranian congress of radiology (ICR) and the 15th congress of Iranian radiographic science association (IRSA). 